Response to Commentaries on The Persecuting God and the Crucified Self: The Case of Olav and the Transformation of His Pathological Self-Image

\title{
Reflections on Olav's Therapy: The Roles of Religious Experience, Self Psychology, and Mentalization
} \author{
GRY STÅLSETT $^{\mathrm{a}, \mathrm{c}}$, LEIF GUNNAR ENGEDAL $^{\mathrm{b}}$, \& ARNE AUSTAD ${ }^{\mathrm{a}}$ \\ ${ }^{\text {a }}$ Modum Bad Psychiatric Center, Vikersund, Norway \\ ${ }^{b}$ MF Norwegian School of Theology, Oslow, Norway \\ ${ }^{\mathrm{C}}$ Correspondence concerning this article should be addressed to Gry Stålsett, Modum Bad Psychiatric Center, \\ Vikersund, Norway, 3370 \\ Email: grysf@online.no
}

\begin{abstract}
Commentaries on the case study of Olav (Stålsett, Engedal \& Austad, 2010) by Richards (2010), Jones (2010), and Malitzky (2010) identify and discuss a number of key issues associated with the case study, including the study's scientific value, the importance of religious experience in relation to psychopathology and psychotherapy, and the roles of selfpsychology, attachment, and mentalization in the therapy change process. In our response to the commentaries, we address these issues. We conclude that all of the perspectives reviewed help to explain the factors that contributed to Olav's success in our VITA treatment program. This multi-perspectival approach was supported by one of the core principles of VITA: the creation of a "culture of inquiry," which specifically helped Olav to experience and regulate his intense negative affects and to understand and integrate his dialectically opposed visions of God and spirituality from a variety of points of view and within a diversity of therapeutic activities.
\end{abstract}

Key words: religious experience; spirituality; the rule of abstinence; representations of God; mentalization; attachment; self psychology; object-relations theory; affect organization; affect regulation; affect integration

\section{BASIC RESEARCH QUESTIONS}

According to Elliott (2002) the main questions in psychotherapy research should be (1) has this client actually changed? (2) Is psychotherapy generally responsible for change? and (3) What specific factors (within and/or outside the therapy) are responsible for change? Richards (2010) addresses the need for bridging the research-practice gap and highlights that methodologically rigorous case studies contribute to evidence-based practice. He underlines the "growing recognition of the limitations of RCTs [randomized clinical trials]"(p, 103) and raises the question of whether they "truly represent a gold standard for obtaining best empirical evidence” (p. 102). We agree that the supposed strengths of RCTs - their scientific control—can turn out to be their weakness, since this control requires the creation of artificial conditions that are not representative of clinical practice (see Leichsenring, 2004). On the other hand, there has long been a need for advanced guidelines for how to present single case 
studies that can build a bridge between research and clinical practice, and between different traditions in the research culture by mixing methods to enhance the internal validity (Kazdin, 1981). According to the three commentators it seems that in writing a case study following this PCSP journal's guidelines (Fishman, 2005) we found a format to present our extensive material in a systematic and rigorous manner.

The commentaries by Richards (2010), Jones (2010), and Malitzky (2010) agree that Olav has truly changed and that it seems more than plausible that treatment is responsible for the changes that have taken place. Thus, the two first questions posed by Elliott seem to be answered positively. But what specific factors are responsible for change? This is a more difficult question to answer simply and will be the focus of the discussion below.

\section{RELIGIOUS EXPERIENCE IN PSYCHOTHERAPY: POSSIBILITIES AND LIMITATIONS}

The case of Olav demonstrates the importance of including religious and existential issues in psychotherapy when these issues seem intertwined in a patient's psychopathology. Central questions are in what way and by what means we address religious issues within the framework of psychodynamic psychotherapy. Richards (2010) and Jones (2010) comment on these questions from different perspectives. Richards refers to what he calls "spirituallyoriented treatment approaches" in which therapists "incorporate spiritual interventions into their practices" (pp. 104). Jones, on the other hand, is convinced that "religious beliefs and practices are not invoked as treatment modalities or psychotherapeutic interventions” (p. 111).

The therapeutic philosophy of the VITA program recognizes these questions as fundamentally important. As documented in the case of Olav, we advocate a certain restrictive line of argument and practice. Consequently, we do not see spiritual or religious practices as integrated parts of the treatment model. We focus on what psychological functions religious beliefs and practices serve for the patient when they occur inside or outside therapy (Stålsett et al., 2010, p. 53). We advocate the classical therapeutic virtue of "abstinence" (see Killingmo, 1997), which here means that the therapist does not convey his own belief, or pray, or give in to the urge to influence the patient in a particular spiritual direction. Our basic position is to foster a "a culture of inquiry" (Stålsett et al., 2010, p. 55) in which it is crucial to facilitate the patient's own investigation into the ambiguities, conflicts, burdens, and resources of his/her religious experiences.

Of special significance here is the focus on "the importance of affect in the psychodynamic inquiry into beliefs and relationships and how affects are connected to ultimate concerns of life” (Stålsett et al., 2010, p. 55). Thus, to foster a culture of inquiry means to investigate and uncover dynamically meaningful configurations in the patient's life story, including religious and existential influence. For this reason and with reference to our current rather diverse spiritual climate, it may be misleading to present or describe our treatment program as “a spiritually-oriented treatment approach” in line with Richards' framing above. Rather, we are giving "the psychological dimension primacy" in the framework of psychotherapy (Sperry \& Shafranske, 2005, p. 23-24), and on this basis focus on spiritual and religious dimensions of human experience. Religious interventions, like prayer, sermons, confession, or other rituals are made available for the patients by pastoral counselors. However, the use this service is the patient's choice. The pastoral counselor may, 
with informed consent from the patient, work in collaboration with the therapeutic staff, in a common understanding of the different tasks and purposes of a psychotherapeutic culture, on the one hand, and pastoral/spiritual care, on the other. Such collaboration was important in the Olav's case for integrating transformed inner representations of self and God, which also seemed to have opened a new access to healthy spirituality and religious belief in Olav.

\section{SELF PSYCHOLOGY, ATTACHMENT, AND OBJECT RELATIONS THEORY}

Jones (2010) states that a self-psychological perspective might suggest an additional reason why the VITA program succeeded where others failed. He states that the treatment program provided an ideal therapeutic matrix with empathic and affirming relational experiences that "the patient can then internalize and use to recreate a more cohesive and efficacious sense of self.” (p. 113). Jones suggests that the empathic/affirming approaches in combination with "a vigorous interpretative stance" are of equal importance for the successful outcome for this patient. In response to this statement, we add that the interpretative analytic interventions were of special importance (as Jones also pointed out) "to unveil resistances, and to explore fantasies in terms of which the patient has reinterpreted reality" (Killingmo, 1997). An example is Olav`s repetitive clinging relationships to women as pain-avoiding behavior (Stålsett et al., p. 58). Without the persistent therapeutic work on his resistance we think that the empathic, affirming position might have ended up gratifying his narcissistic needs, hampering his transformation that seemed facilitated by working directly with Olav's abandonment feelings.

The reason why we highlight object-relations theory more than self psychology is our view of God as an object representation that is affectively connected with inner representations of parents. This is an understanding that probably would not be addressed in the same way in a purely self-psychology paradigm that focuses on the self rather than on object-relational transference processes. In the field of psychology of religion, we now believe that object-relations theory captures more of the depth of inner psychic drama. Objectrelations understanding seems particularly useful in understanding paranoid dynamics. This relates to what we have learned from William Meissner (1996): the therapist should have the same attitude towards a religious patient as toward a paranoid one. Thus, just as with a paranoid client the therapist should not start a discussion about whether the patient is persecuted and haunted or not, with a religious patient the therapist should not start a discussion about whether God exists or not. Rather, the therapist should focus on the psychological function and meanings of the target paranoid or religious beliefs. Of note here is Malitzky's (2010) observation that Olav did not only have religious inner representations, but he also suffered from paranoid dynamics.

On the other hand, we agree with Jones ( 2010) that self-psychological concepts are useful in cases like Olav's, with religious objects viewed as self objects (see Rector, 2000). For example, we would say that Olav had a twinship self-object (Rector, 2000) in Jesus, as Olav stated in an explanation the drawing in Figures 5 of Stalsett et al. (2010), "The crucified little child, nailed to the big blue cross is me” (p. 64). Regardless of theory it is of utmost importance to facilitate a climate which is safe, empathic, and affirming enough to guarantee that emotional arousal-like rage or shame-can be tolerated and addressed, so that patients can let go of strict censorship around expressing their feelings. 


\section{ATTACHMENT AND MENTALIZATION}

Malitzky`s commentary (2010) expands the theoretical reflection on the case study of Olav by connecting the VITA treatment model to the concept of "mentalization," that is, a focus on mental states in oneself and others, and "symbolization," described as "a fluency in mental state language” (Bateman and Fonagy, 2004). We find Malitzky's comments very relevant, highlighting Olav`s enhanced mentalization capacity as the most effective mechanism behind the dynamic changes. Referring to Fonagy, Gergely, Jurist, \& Target (2002) on insecure and disorganized attachment as strongly associated with low levels of mentalization, Maltizky comments on Olav`s developmental failure. Malitzky mentions the death of Olav's twin brother, leading to Olav's mother's depression in his early childhood; his father's idealizing of the dead twin brother and related high moral expectations placed on Olav; and the parents' divorce in Olav's late adolescence, creating a rigid, defensive set of split self-object representations. The confrontation with failures in Olav's own life led to psychic decompensation to what we might characterize as a borderline level of psychic functioning, although the clinical picture also was more complicated, including major depression with psychotic features and comorbid paranoid personality disorder and narcissistic traits (Stålsett et al., 2010, p. 91).

This corresponds well with one of our theoretical frames of reference understanding borderline personality disorder as a developmental arrest of the real self, building a "false self” as a defense against emotional abandonment (Masterson, 1988). According to Masterson the borderline syndrome is characterized by serious depression over abandonment, with six distinctive emotional components: sadness, panic, rage, guilt, helplessness (hopelessness), and emptiness (void), named as "The Six Horsemen of the Psychic Apocalypse” to illustrate the inner terror of the abandonment depression (Masterson, 1976, p. 61). Within the therapeutic culture of inquiry in the VITA program, Olav was challenged to look into his own clinging and dependent behavior to see how it served a defensive function. Gradually through confronting emotions like anger, sadness, shame, and guilt, which overwhelmed him as soon as he was on his own, Olav could see his own pattern and how his feelings of loneliness fuelled new resistance and pain-avoiding behavior. This was especially anxiety provoking since it sounds just like what Malitzky so precisely formulated: "an environmental failure led Olav to a defensive retreat from reflective functioning” (p. 121). Through hard work against his own resistance and anxiety, Olav increased awareness, did emotional diary writings, and verbalized his existential concerns and his "unacceptable” affects (Smyth,1998; Rizzuto, 2003). This gradually seemed to contribute to a reorganization of the way in which Olav's affects were connected to inner objects (Stålsett et al., 2010), which in turn led to an increased ability to regulate affect and to mentalize during the course of treatment (Schore, 1994, 2003; Fonagy \& Target, 1998; Fonagy et al., 2002).

During treatment Olav was able to integrate into his experience his earlier criminalized, forbidden affects (prohibited from experience or expression by Olav's creations of The Committee and the Persecuting God). Olav was also able to integrate new experiences of "being Olav," which were perceived and recognized as his "real self," in accordance with his articulated goal for treatment. As Olav's self-representation was transformed, it opened the possibility of a transformation of his God representation from a split-off, projectiveintrojective object to a God as a transitional object available for healthy object use (Malitzky, 2010, p. 123). This corresponds to Ana-Maria Rizzuto`s theory of the development and 
psychic function of the God representation, constituting one of the main theoretical cornerstones of the VITA model (Rizzuto, 1979).

Our understanding of a developmental separation failure and arrest underlying Olav's pathological self structure may in many ways connect to attachment theory. We are aware of the controversy between attachment theorists and object-relation theorists concerning projections, fantasies, and defense mechanisms (Granqvist, 2006; Rizzuto, 2006 ).When "attachment figures" replace "object representations," we may lose the unconscious contributions to the inner drama (such as "Oedipal dynamics" and other affects and fantasies related to parents). In our opinion it is crucial to have this understanding of distortions in perception of self and others conveyed by the level of differentiation in the individual's language when talking about affects and relationships (Rizzuto, 2003). Our understanding of mentalization is given and further developed in our case study:

to make the patient aware of relational patterns and transference reactions, and the subjectivity of their perceptions; to trace emotional conflicts to the "right address" so feelings are not acted out inappropriately towards the wrong person; [and] to alter destructive interpersonal responses, e.g., projections, submissiveness, and overconscientiousness due to shame and guilt (Stalsett et al., 2010, Table 1, p. 85).

This view seems to be in line with Malitzky`s (2010) discussion of mentalization. He states that in an expanded understanding, "nearly all of the treatment strategies and components in the VITA model can be understood with mentalization in mind" (p. 121). Malitzky believes in the importance of psychoeducation in affect work and psychotherapy, and we feel he has captured the essence of the crucial elements in the VITA model in his elaboration of the mentalization concept. We understand what he says in this context and agree when he says that "work with existential/religious concerns within the framework of enhanced mentalization was the mechanism underlying therapeutic effectiveness in this case" (Malitzky, 2010, p. 124).

We believe there is a connection between Olav`s insecure and disorganized attachment and his lack of mentalization capacity. This is illustrated by his oscillation between what Bateman ad Fongy (2004) call the "psychic equivalence mode" and the "pretend mode" of functioning (Malitzky, 2010, p. 119). What Masterson ( 1988) called "lacking the capacity to tolerate and endure the abandonment depression” might be understood as a lack in mentalizing capacity due to attachment failures. This theoretical perspective can account for the importance of creating trust and acceptance of Olav`s psychic reality as it was represented in his religious symbolisation. Although he was tortured by The Committee, it represented "a minimum amount of relatedness" (Rizzuto, 1979), an ultimate kind of attachment being better than experiencing emptiness and void. Olav's process of obtaining a more integrated object relation to God, allowing a benevolent God "to be there for him," was preceded by using the Modum Bad Psychiatric Center as a "safe haven” for developing a secure attachment to his therapists, making his developing mentalization capacity possible. This implied reorganization of Olav's affects connected him to his inner objects; integrated his earlier, unacceptable affects; and increased his affect regulation through an increased mindfulness and meta-reflection. The focus on Olav's affects appeared clinically crucial and in line with contemporary research, which indicates that facilitation of patient affective experience and expression is associated with patient improvement over the course of psychodynamic psychotherapy ( Diener, Hilsenroth, \& Weinberger, 2007) 
Malitzky (2010) points out that the assessment battery in our VITA program would be enhanced by using Steele and Steele's (2008) Adult Attachment Interview or AAI, which is based on a theoretical framework consistent with the psychoanalytic, object-relations perspective that we use. When Olav's treatment took place (1999-2000), the AAI was not available to us. We d plan to use the AAI in a new process study of the VITA treatment model currently being conducted.

\section{CONCLUSION}

We appreciate the thoughtful, perceptive, insightful, and scholarly nature of the three commentaries on our case of Olav. As a whole, they present a range of theoretical perspectives that enhances our understanding of the transformation process in Olav's case. Together we believe that they can speak to a wide audience of clinicians and researchers. Transformational change is understood as a life-defining experience demarcating a period between an old self and a new self, often building on an agonizing conflict (White, 2004, p. 421). In Olav's case the transformational experience was distinct from a sudden religious experience transforming the person in the here and now. Rather, Olav's transformation experience was facilitated by a systematic, planned psychotherapeutic intervention that involved a deconstruction of pathological meaning construction, and enhanced affect reorganization and regulation in the framework of development of "enhanced mentalization," to put it in Malitsky`s terms. In other words, as we describe in Olav's case study (Stalsett et al., 2010), the transformation of Olav's pathological self-image can be understood in terms of the development of in Olav of the capacities for mindfulness, mentalization, and metareflection that assisted him in in-depth work with his affect awareness and regulation and his views of the role of religion and existential themes in his life.

The process of Olav's issues of transformation are addressed in all three commentaries as connected to changes in self- and object representations. Jones refers to remarkable changes in Olav's drawings, interpreted as signs of profound personality and cognitivedevelopmental shifts. Malitzky points to transformation of the bad, internal object of the harsh Committee into a positive, internal representation, and notes how Olav's representation of God in his drawings changed. Richards mentions how the transformation of inner representations of self, parents, "The Committee," and God "facilitated his emotional and spiritual healing and maturation” (2010, p. 105). The three commentaries correspond with our formulation that "the transformation of the God image and the harsh Committee from abusive to affirming was the crucial therapeutic work to heal Olav`s 'Crucified Self'" (Stålsett et al., 2010, p. 78). We agree with Malitzky that the transformational processes are dependent on mentalization of pathogenic representations. This is mediated through "the spoken word" within the context of a respectful and interpretive transferential dialogue between patient and therapist, "the essential element in bringing about the transformation of the subject and the elimination of symptoms“ (Rizzuto, 2003).

A general question in the psychotherapy research field is, " How do people change, and how can we change to help many more people?” (Prochaska, 1999). Illustrated in our experience with Olav, we believe that one way of answering these questions is to employ a psychotherapeutic theoretical framework that facilitates transformation from several angles at the same time, with the goal of loosening up rigidity, the common trait of psychopathology in thought, affects, inner representations, behavior, and perspectives for the future. This focus on 
loosening up rigidity connects to the domain of spiritual and religious belief as well, and because such beliefs often have a particularly strong impact on the inner dynamics, it is particularly crucial to use psychotherapeutic insights and knowledge to address religious and spiritual issues. Stiles, Honos-Webb, and Surko (1998) discuss the importance in successful therapy for patient and clinician responsiveness to each other. We agree and have striven to create in VITA a therapeutic culture and framework where the generality of theories and clinical understanding can be tailored to the specific patient, addressing and giving significance in "a culture of inquiry" to his or her inner world including God, existing or not, persecuting or benevolent.

\section{REFERENCES}

Bateman, A. \& Fonagy, P. (2004). Psychotherapy for borderline personality disorder: Mentalization based treatment. Oxford, UK: Oxford University Press.

Diener, Hilsenroth \& Weinberger, (2007). Therapist affect focus and patient outcomes in psychodynamic psychotherapy: A meta-analysis. American Journal of Psychiatry,164, 936-941.

Elliott, R. (2002) Hermeneutic single-case efficacy design. Psychotherapy Research 12, 1-21.

Fishman, D. B. (2005). Editors`s introduction to PCSP_-from single case to database: A new method for enhancing psychotherapy practice. Pragmatic Case Studies in Psychotherapy, Vol. 1(1), Article 2, 1-50: Available: http://hdl.rutgers.edu/1782.1/pcsp_journal

Fonagy, P., Gergely, G., Jurist, E. L., \& Target, M.(2002). Affect regulation, mentalization and the development of the self. New York, NY: Other Press

Fonagy, P. \& Target, M. (1998). Mentalization and the changing aims of child psychoanalysis. Psychoanalytic Dialogues, 8, 87-114.

Granqvist,P. (2006): On the relation between secular and divine relationships: An emerging attachment perspective and a critique of the "depth" approaches. The International Journal for the Psychology of Religion, 16, 1-18.

Jones, J.W. (2010). psychotherapeutic change and spiritual transformation: The interaction effect. Pragmatic Case Studies in Psychotherapy, Vol. 6(2), Article 3, 109-117: Available: http://hdl.rutgers.edu/1782.1/pcsp_journal

Kazdin, (A.E.) 1981).Drawing valid inferences from case studies. Journal of Consulting and Clinical Psychology, 49, 183-192

Killingmo, B. (1997). The so-called rule of abstinence revisited. Scandinavian Psychoanalytic Review, 20, 144-159.

Leichsenring, F. (2004). Randomized controlled versus naturalistic studies: A new research agenda. Bulletin of the Menninger Clinic, 68, 137 -151

Malitzky, M. S. (2010). Dissolution of "The Committee": Mentalization and psychic transformation in the case of Olav. Pragmatic Case Studies in Psychotherapy, Vol. 6(2), Article 4, 118-125: Available: http://hdl.rutgers.edu/1782.1/pcsp_journal

Masterson, J. F. (1976). Psychotherapy of the borderline adult: A developmental approach. Bristol, PA: Brunner/Mazel

Masterson, J. F. (1988). The search for the real self: Unmasking the personality disorders of our age. New York: The Free Press

Meissner, W.W. (1996). The pathology of beliefs and the beliefs of pathology. In Shafranske E.P. (Ed.), Religion and the clinical practice of psychology. Washington D.C.: American Psychological Association, 241-267. 
Prochaska, J.O. (1999) How do people change, and how can we change to help many more people: In: Hubble, M.A., Duncan, B.L. \& Miller, S.D. (eds) The heart and soul of change: What works in therapy? Washington DC: APA Books

Rector,L.J. (2000): Developmental aspects of the twinship self-object need and religious experience. In: A.I. Goldberg (ed). Progress in self psychology, vol.16, 257-276. Hillsdale, N.J: The Analytic Press.

Richards, P.S. (2010) The role of spirituality in Olav`s treatment and recovery: Commentary on an exemplary case report. Pragmatic Case Studies in Psychotherapy, Vol. 6(2), Article 2, 101-108: Available: http://hdl.rutgers.edu/1782.1/pcsp_journal

Rizzuto, A.M. (1979). The birth of the living God. Chicago: University of Chicago Press.

Rizzuto, A.M. (2003). Psychoanalysis: The transformation of the subject by the spoken word. Psychoanalytic Quartly, LXXII, 287-323.

Rizzuto, A.M. (2006). Discussion of Granqvist`s article on "The relation between secular and divine relationships: An emerging attachment perspective and a critique of the 'depth' approaches." The International Journal of Psychology of Religion, 16, 19-28.

Schore, A.N. (1994). Affect regulation and the origin of the self. Hillsdale, N.J: Erlbaum.

Schore, A.N.(2003). Affect dysregulation and disorders of the self. New York; London:W.W. Norton \& Company.

Smyth, J. M. (1998) Written emotional expression: effect size , outcome types, and moderating variables. Journal of Consulting Clinical Psychology, 66, 174-184.

Sperry, L \& Shafranske,E.P. (Eds.). (2005). Spiritually oriented psychotherapy. American Psychological Association. Washington, D.C.

Stiles, W.B., Honos-Webb, L. \& Surko; M. (1998). Responsiveness in psychotherapy. Clinical Psychology: Science and Practice, 5, 439-458.

Steele, H. \& Steele, M. (2008). Clinical applications of the Adult Attachment Interview. New York, NY: The Guilford Press.

Stålsett, G., Engedal, L.G., \& Austad, A. (2010). The persecuting God and the crucified self: The case of Olav and the transformation of his pathological self-image. Pragmatic Case Studies in Psychotherapy, Vol. 6(2), Article 1, 49-100: Available: http://hdl.rutgers.edu/1782.1/pcsp_journal

White, W.L. (2004). Transformational change: A historical review. Journal of Clinical Psychology, 60, 461-470. 